\title{
Ultrafast Laser Patterning of OLEDs on Flexible Substrate for Solid-state Lighting
}

\author{
Dimitris KARNAKIS*, Andrew KEARSLEY and Martyn KNOWLES \\ Oxford Lasers Ltd, Unit 8 Moorbrook Park, Didcot, Oxfordshire OX11 7HP, UK \\ E-mail: dimitris.karnakis@oxfordlasers.com
}

\begin{abstract}
Rapid developments in the organic LED technology on flexible foils promise to deliver thin, lightweight and power-efficient light sources for intelligent lighting applications. Laser patterning of OLEDs on glass for display applications has been widely reported. However, fewer reports discuss patterning of OLEDs on flexible substrates, which presents a novel optical engineering challenge. Our aim here is to quantify the potential of picosecond lasers for this application and determine a robust laser process window for large area selective OLED electrode patterning. This should involve the complete removal of (i) $130 \mathrm{~nm}$ thick ITO anode on a barrier layer stack and (ii) $100 \mathrm{~nm}$ thick Ba/Al cathode on $180 \mathrm{~nm}$ thick LEP/PEDOT:PSS active organic layers while leaving intact all underlying layers. Detailed laser ablation studies at 532 and $355 \mathrm{~nm}$ reveal that ps lasers uniquely facilitate roll-to-roll OLED manufacturing by providing fine resolution. Damage thresholds, process quality and process speed limitations will be discussed. Careful crater examination reveals that the choice of laser pulse duration is more important than wavelength for a given layer thickness. A photomechanical stress-induced ablation mechanism is believed to be key for this process and debris-free, low temperature patterning can be achieved.

DOI: $10.2961 / \mathrm{jlmn} .2009 .03 .0014$
\end{abstract}

Keywords: OLED, ITO, thin-film patterning, picosecond laser ablation, stress-assisted ablation

\section{Introduction}

Rapid developments in OLED technology are beginning to make significant inroads to the commercial world [1] with OLED displays already available in certain niche applications. Lasers are typically used in OLED manufacturing for patterning the active layers or the transparent oxide and metal thin-film electrodes [2,3]. Research has even shown that lasers can be used to deposit OLED layers on demand via a direct-write dry printing method called laser-induced forward transfer (LIFT) [4].

Commercial interest is now growing in producing large area OLEDs on plastic foils for intelligent solid-state lighting [5]. These roll-to-roll manufactured foils promise to deliver thin, lightweight and power-efficient light sources for general, architectural or automotive lighting and signage applications. However, most flexible plastic substrates are permeable to water and oxygen and cannot provide adequate OLED encapsulation. To avoid severe device degradation, a transparent thin-film stack of several organic/inorganic barrier layers must be incorporated between the OLED films and the plastic substrate (Figure 1) [6]. Manufacturing these barrier layers represents a great challenge. Selective laser patterning therefore of the thermally thin OLED films atop such barrier layers is even more demanding, as any laser structuring must leave them totally unharmed. Additionally any contamination from laser-generated debris is severely prohibited to avoid longterm degradation of the OLED device performance and lifetime.

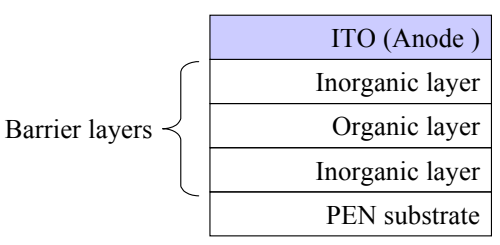

(a)

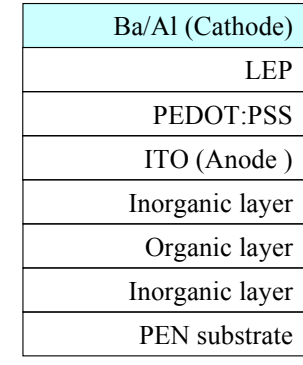

(b)
Fig. 1 Multilayer OLED stack on PEN substrate. Laser patterning of (a) the anode layer on a barrier stack and (b) the cathode layer on the light-emitting layer (LEP) is necessary.

In other words, laser ablation must proceed via a low temperature removal mechanism suppressing any thermal characteristics that could potentially damage the OLED or its multilayered substrate. This is preferably accomplished using ultrafast lasers via a stress-assisted film fracture and ejection (spallation) ablation mechanism rather than involving vaporization or other phase change.

Our aim [5] is to quantify the potential of picosecond lasers for patterning a $130 \mathrm{~nm}$ thick ITO film on the barrier layer and a $100 \mathrm{~nm}$ thick cathode bilayer $(\mathrm{Ba} / \mathrm{Al})$ on the organic light emitting layer (LEP) and ideally determine a process window for large area selective OLED patterning. 


\section{Selective laser patterning of OLEDs by stress- assisted ablation}

For stress-assisted ablation to occur both the thermal and stress confinement criteria must be fulfilled in the absorbing layers [7]. This means that (a) maximum light absorption in the film is required with minimum thermal conduction losses away from the irradiated area and (b) rapid heating to produce very high transient thermoelastic stress to exceed the film fracture limit before any thermal expansion allows for stress relaxation. Therefore both laser wavelength and pulse duration seem critical.

The characteristic times to consider further are: (i) the thermal relaxation time $\tau_{\text {th }}$ i.e. the time needed to dissipate heat generated by the laser pulse, given by $\tau_{\mathrm{th}}=\delta^{2} / 4 \chi$ and (ii) the acoustic relaxation time $\tau_{\mathrm{ac}}$, i.e. the time needed to initiate a collective motion of atoms or molecules within the absorbing volume, given by $\tau_{\mathrm{ac}}=\delta / \mathrm{C}_{\mathrm{s}}$. Here $\delta$ refers to the smallest dimension of the heated volume, typically the laser or thermal penetration depth assuming a much larger laser spot size, $\chi$ is the film thermal diffusivity and $C_{s}$ is the sound speed in the film.

If the laser pulse duration $\tau$ is shorter than the thermal relaxation time $\tau_{\text {th }}\left(\tau<\tau_{\text {th }}\right)$, the thermal confinement criterion is fulfilled maximising the temperature in the heated volume by restricting thermal diffusion to the substrate layers and efficiently generating thermoelastic stress. Additionally, if the laser pulse duration is shorter or comparable to the characteristic acoustic relaxation time $\tau_{\mathrm{ac}}$ $\left(\tau<\tau_{\mathrm{ac}}\right)$, laser heating of the target will proceed at nearly constant volume. The heated material will have little time to expand and the large temperature gradients will cause a buildup of high compressive stresses. The interaction of the laser-induced compressive stresses with the free surface of the irradiated sample can then result in generation of tensile stresses, sufficiently high, that can overcome the dynamic strength of the material causing mechanical fracture or promote cavitation and fragmentation in a metastable liquid [8]. With this approach differences in thermal expansion between the stack layers can be neglected as the strain is assumed negligible.

Since ITO absorbs weakly at $355 \mathrm{~nm}$ with a laser penetration depth of $\sim 2.4 \mu \mathrm{m}\left(\alpha_{355}=4100 \mathrm{~cm}^{-1}\right)$, uniform film heating is assumed through the entire film along the beam propagation. Taking $\delta=130 \mathrm{~nm}$, results in thermal and acoustic relaxation times of $2 \mathrm{~ns}$ and 43 ps respectively as shown in Table 1. This means that a laser pulse shorter than $\sim 43$ ps, will satisfy both the thermal and stress confinement criteria for stress-assisted ablation of ITO restricting thermal diffusion to the barrier layers and

Table 1 Estimated thermal and acoustic relaxation times for 10 ps laser irradiated OLED thin-film electrodes. Film thickness; Anode (130nm), Cathode (100nm)

\begin{tabular}{cccccc}
\hline Material & $\begin{array}{c}\delta \\
(\mathrm{nm})\end{array}$ & $\begin{array}{c}\chi \\
\left(\mathrm{cm}^{2} / \mathrm{sec}\right. \\
)\end{array}$ & $\begin{array}{c}\mathrm{Cs} \\
(\mathrm{m} / \mathrm{s})\end{array}$ & $\begin{array}{c}\tau_{\text {th }} \\
(\mathrm{ps})\end{array}$ & $\begin{array}{c}\tau_{\mathrm{ac}} \\
(\mathrm{ps})\end{array}$ \\
\hline Anode & 130 & 0.0216 & 3045 & 1950 & 43 \\
Cathode & 72 & 0.9986 & 5300 & 13 & 13 \\
\hline
\end{tabular}

efficiently generating thermoelastic stress in the film. However, as only a small fraction of the incident energy will be deposited in the weakly absorbing ITO, care should be taken to avoid optical damage from direct absorption in the underlying barrier layers by keeping the incident intensity low below the barrier ablation threshold.

The metal cathode bilayer $(\mathrm{Ba} / \mathrm{Al})$ absorbs strongly at $\mathrm{UV}$, visible or IR wavelengths and hence irradiation at 532 $\mathrm{nm}\left(\alpha_{532}=1.2 \times 10^{6} \mathrm{~cm}^{-1}\right)$ is expected to remove the cathode layer while leaving unharmed the transparent LEP layer underneath. The laser heated volume estimation is key for determining the thermal and acoustic relaxation times here. The optical penetration depth is very short at $8.3 \mathrm{~nm}$ and laser heating of the film will thus be dictated by thermal diffusion during the laser pulse. Since the thermal response of metals to ultrafast excitation is a non-equilibrium process with different electron and lattice heating rates, the classical square root dependence of heat diffusion on laser pulse duration does not apply. Our estimate of the thermal diffusion depth based on [9] is $72 \mathrm{~nm}$ using an electronphonon coupling constant for $\mathrm{Al}$ of $3.1 \times 10^{17} \mathrm{~W} / \mathrm{m}^{3} \mathrm{~K}[10]$ and a weaker dependence on laser pulse duration $\tau^{0.2}$. With $\delta=72 \mathrm{~nm}$ for a $10 \mathrm{ps}$ laser pulse, both thermal and acoustic relaxation times are approximately equal at $\tau_{\mathrm{th}} \approx \tau_{\mathrm{ac}}=13 \mathrm{ps}$.

\section{Experimental}

Both laser sources used here are commercially available DPSS mode-locked Nd: $\mathrm{YVO}_{4}$ amplifiers emitting laser pulses of 10 ps (FWHM) duration. For ITO patterning an Oxford Lasers Picolase 1000 micromachining system was used at $355 \mathrm{~nm}$. The laser repetition rate was fixed at $10 \mathrm{kHz}$ and the beam was focused with an f-theta telecentric lens $(f=100 \mathrm{~mm})$ to an estimated spot size of 32 $\mu \mathrm{m} \varnothing$. The focussed spot was translated across the ITO surface using a galvanometer scanner with maximum translation speed of $2 \mathrm{~m} / \mathrm{s}$. The sample consisted of a 130 $\mathrm{nm}$ thick ITO layer sputtered on a multilayered barrier stack of alternating PECVD deposited SiN and organic planarisation layers with approximate thicknesses of $300 \mathrm{~nm}$ and $40 \mu \mathrm{m}$ respectively. These were deposited on a $100 \mu \mathrm{m}$ thick PEN foil. All layers underneath the ITO were transparent between $400-800 \mathrm{~nm}$. The $\mathrm{SiN}$ absorption coefficient at $355 \mathrm{~nm}$ is estimated as $\alpha=1.35 \times 10^{4} \mathrm{~cm}^{-1}$.

For cathode patterning a Coherent Talisker laser was used at $532 \mathrm{~nm}$. A similar beam delivery setup was utilised to focus the beam to a $35 \mu \mathrm{m}$ diameter spot. Single or multiple pulse ablation tests were conducted by controlling the incident number of laser pulses per area. The cathode sample consisted of a metal bilayer (thickness: Al $100 \mathrm{~nm}$, Ba $5 \mathrm{~nm})$ thermally evaporated on the OLED layers $(80 \mathrm{~nm}$ thick white emitting polymer LEP (Merck) and $100 \mathrm{~nm}$ thick conductive Poly 3,4-ethylenedioxythiophene polystyrenesulfonate (PEDOT: PSS) Agfa Orgacon). The OLED layers were slot-die coated on the ITO anode. All layers underneath the cathode were transparent at $532 \mathrm{~nm}$.

Ablation took place in air and the etched layers were vacuum extracted above the target. In both cases, the pulse energy was varied using a half-waveplate and polarizer attenuation setup. The ablated samples were investigated with an optical microscope or white light interferometer. 


\section{Results and discussion}

\subsection{Laser patterning of ITO on barrier layers}

By varying the input pulse energy and scanner speed, a range of incident laser fluences at different pulse overlaps was examined for ITO line scribing. The average fluence ablation threshold at $355 \mathrm{~nm}$ for single-pulse ablation was determined as $50-55 \mathrm{~mJ} / \mathrm{cm}^{2}$. Below this value no ablation was recorded. At fluences between $75-130 \mathrm{~mJ} / \mathrm{cm}^{2}$ ITO ablation was also accompanied by surface roughening of the underlying SiN barrier layer.

Figure 2 shows scribed lines in ITO at $62 \mathrm{~mJ} / \mathrm{cm}^{2}$ just above threshold, using one (fig.2a) or four (fig.2b) overlapping pulses per area. Non-continuous lines of discrete irregular shaped craters were obtained in both cases, seemingly elongated in the direction of scanning. This implies that the ablation threshold was only exceeded locally by a very narrow intensity part near the tip of the focused Gaussian beam. The ablated films appear to have been removed intact with no damage to the underlying SiN. There is also no evidence for melting or any other phasechange and by and large, no debris was found scattered in or around the craters, suggesting that ITO and the barrier layers have probably experienced very low temperature rise.

The fractured morphology of the craters reveals clearly the photomechanical character of ablation in this case. This is particularly evident near the crater edges where partially ablated ITO was found as lifted off flakes, which were still attached to the parent film and clearly not thermally decomposed. In some cases also, very few unsuccessfully extracted ablated layers, were located nearby the craters as softened curled up flakes, supporting this view further.

By adjusting the scribing speed the ablated craters can be joined to form an uninterrupted continuous scribed line on ITO. Depth profile measurements of the scribed line (Fig.3) confirm clean ablation with smooth uniform floors, no raised edges in the adjacent unexposed areas and an etched depth of $123 \mathrm{~nm}$ indicating that only ITO has been

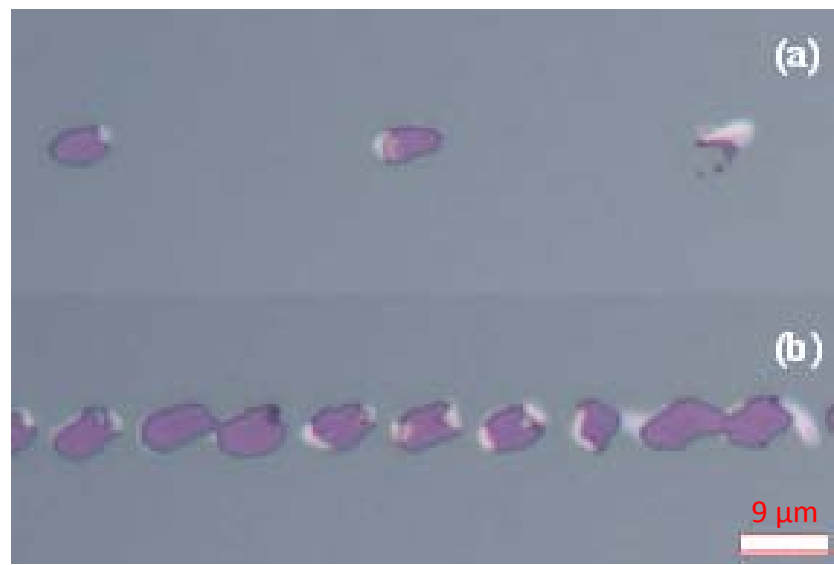

Fig. 2 High magnification (500x) optical images of 10ps laser scribed ITO (grey) on barrier stack (purple) for OLED fabrication. ( $355 \mathrm{~nm}, 62 \mathrm{~mJ} / \mathrm{cm}^{2}$ ) (a) one pulse (300 $\mathrm{mm} / \mathrm{s})$ and (b) four pulses per area $(75 \mathrm{~mm} / \mathrm{s})$. The ablated craters are a fraction of the illuminating laser spot on target. Bar denotes $9 \mu \mathrm{m}$
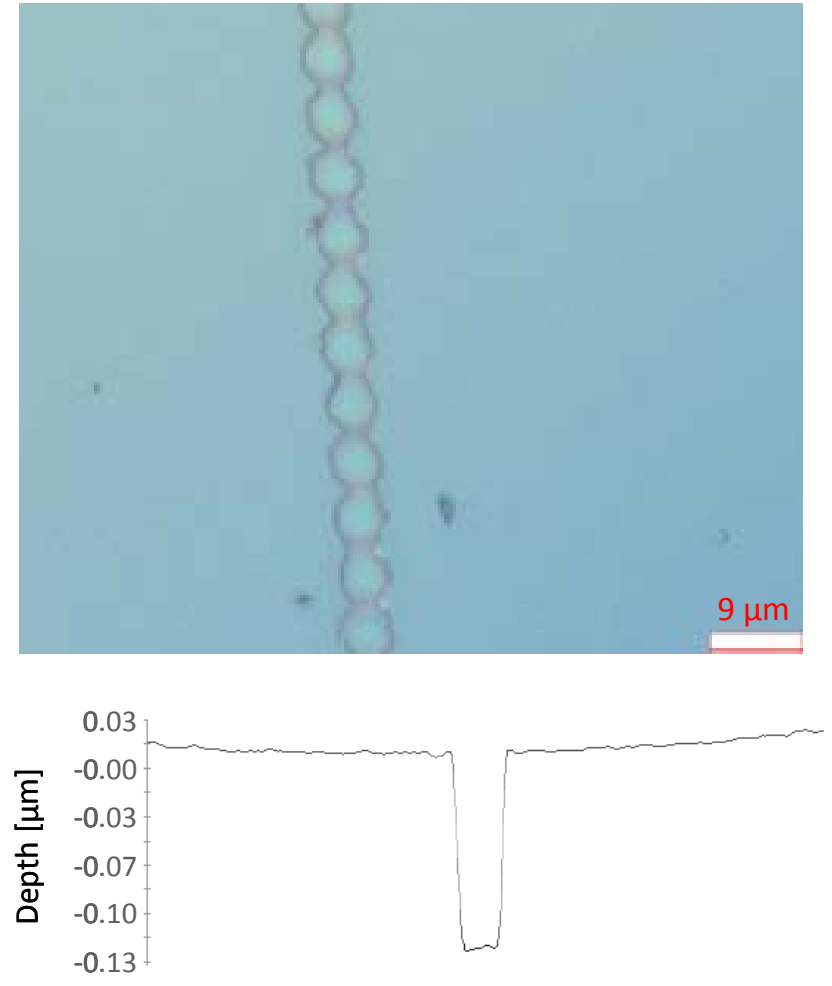

Fig. 3 Optical image of scribed line in ITO using a 355nm $10 \mathrm{ps}$ focused Gaussian beam. Bar $9 \mu \mathrm{m}$. Optical profiler depth measurement of $355 \mathrm{~nm}, 10 \mathrm{ps}$ ablated ITO crater; scribed depth: $123 \mathrm{~nm}$, crater width: $9-12 \mu \mathrm{m},(\mathrm{Y})$ scale: $15 \mathrm{~nm} / \mathrm{div}$

removed from the stack. Furthermore, electrical continuity measurements confirmed that such lines could be used to create electrically isolated OLED tiles.

Very similar ablation results, not reported here, have been preliminary achieved with IR (1064 $\mathrm{nm})$ and visible (532 nm) picosecond lasers $(\tau=10 \mathrm{ps})$ incident, either directly on the ITO surface or through the transparent foil, suggesting that ITO patterning on a barrier stack shows limited wavelength dependence.

Although the adhesion strength between films in the current stack was not measured, overcoming ITO adhesion to the barrier layer is expected to be undemanding in the order of few tens of $\mu \mathrm{J} / \mathrm{cm}^{2}$ [11]. In photomechanical ablation, small fractions of the absorbed laser energy go into overcoming film adhesion to the substrate and mostly go into elastic stress energy. A portion thereof is used into plastic deformation of the target, generation of internal free surfaces (fracture by generation of cracks or voids), and kinetic energy of the ejected material. Negligible energy goes into a phase change, except some evaporation into voids and cracks [7]. This is in contrast to fracture due to an explosive phase change when the driving force is the pressure in expanding vapor bubbles.

That agrees well with the experimental findings here and is in line with energy balance considerations. The input energy load $\mathrm{E}_{\mathrm{L}}=(1-\mathrm{R}) \alpha \mathrm{F}_{\mathrm{P}}$ at threshold in ITO is estimated as $436 \mathrm{~J} / \mathrm{cm}^{3}$ using a thin-film stack reflectivity $\mathrm{R}$ $=3.2 \%$ at $355 \mathrm{~nm}, \alpha=4100 \mathrm{~cm}^{-1}$ and peak fluence $\mathrm{F}_{\mathrm{P}}=$ $0.11 \mathrm{~J} / \mathrm{cm}^{2}$. Neglecting any heat losses and with an input load much 
$(\alpha)$

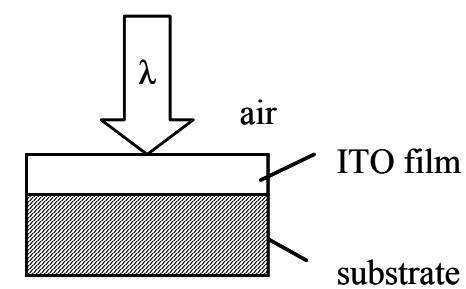

(b)

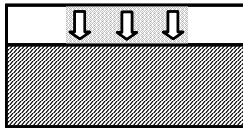

Confined

Heating

(c)

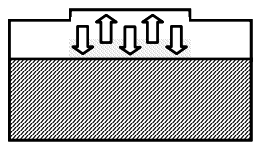

Tensile stress generation

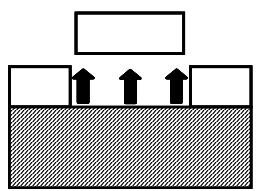

contributing to the overlying ITO ablation. The possibility of expanding vapour originating at the $\mathrm{SiN}$ surface to blow off the ITO layer cannot be excluded either, although no visible surface changes were detected on the barrier layer below $75 \mathrm{~mJ} / \mathrm{cm}^{2}$. Also, as mentioned above, very similar ITO ablation results were recorded with laser irradiation at $532 \mathrm{~nm}$ and $1064 \mathrm{~nm}$ where the SiN is totally transparent. Further experimental evidence investigating this effect microscopically is necessary to clarify the process.

Essentially, since ablation is based on single-pulse removal, process speed limitations are likely to arise from the maximum available laser output and the ability to separate the delivered laser pulses at the workpiece at high repetition rates with an appropriate beam delivery and motion control setup. Also the stress profile in the film is directly related to the gradient of the incident laser intensity profile. Sharper gradients at the spot edges from a reshaped Gaussian beam are expected to provide cleaner boundaries with less delamination. Ca tests are currently in progress to examine the barrier layer integrity from picosecond laser ablation of ITO before proceeding further.

Fig. 4 Schematic representation of stress-assisted ITO film ablation on barrier substrate layer; (a) $t=0$, light absorption, (b) rapid laser heating under thermal and stress confinement, maximum compressive thermoelastic stress (c) thermal expansion relaxation with tensile stress generation, (d) tensile strength exceeded, film fracture and ejection

lower than the melting or vaporisation enthalpies, all absorbed energy will be used to raise the film temperature by $\Delta \mathrm{T}=\mathrm{E}_{\mathrm{L}} / \rho \mathrm{C}$. With ITO density $\rho=7.1 \mathrm{~g} / \mathrm{cm}^{3}$ and specific heat $\mathrm{C}=340 \mathrm{~J} / \mathrm{kgK}$, this is estimated as only $\Delta \mathrm{T}=$ $181 \mathrm{~K}$, well below the melting point of $2100 \mathrm{~K}$. Since ITO is weakly absorbing at $355 \mathrm{~nm}$, it is reasonable to assume the film is uniformly heated across its entire thickness along the beam propagation. The maximum uniaxial thermoelastic compressive stress $\sigma=\mathrm{aE} \Delta \mathrm{T}$ generated in the film under stress confinement (no strain during heating) can then be estimated as $150 \mathrm{MPa}$ with linear thermal expansion coefficient $\mathrm{a}=7 \times 10^{-6} \mathrm{~K}^{-1}$ and Young's modulus $\mathrm{E}=118 \mathrm{GPa}$. Although a definite ablation route is not entirely clear in the absence of more experimental information, it is feasible that a large enough tensile stress component can subsequently develop in the film upon relaxation, which can exceed the ITO tensile strength and cause film fracture and ejection. Reported values for ITO tensile strength of $125 \mathrm{MPa}$ [12] could support this notion.

The role of the substrate cannot be ignored [13] due to the weak ITO absorption, as the SiN layer will absorb part of the transmitted energy. No ablation of the SiN takes place though below $75 \mathrm{~mJ} / \mathrm{cm}^{2}$. Although difficult to approximate the exact input load on that film due to thinfilm interference [14], a significant temperature rise estimated as $\sim 2300 \mathrm{~K}$ could be present at the ITO-SiN interface which is probably approaching the current SiN film melting point. For the present film stack, values of $\mathrm{R}(\mathrm{SiN})=26.6 \%, \alpha=1.35 \times 10^{4} \mathrm{~cm}^{-1}$ and a peak fluence of $0.1 \mathrm{~J} / \mathrm{cm}^{2}$ were used. Such high temperature would certainly cause transient interface displacement and although the thermal expansion of the current $\mathrm{SiN}$ layer matches well that of ITO, it cannot be excluded from partly

\subsection{Laser patterning of $\mathrm{Ba} / \mathrm{Al}$ cathode on light emitting polymer (LEP)}

Single-pulse ablation of the $\mathrm{Ba} / \mathrm{Al}$ cathode bilayer on active OLED layers was investigated using picosecond 532 $\mathrm{nm}$ laser irradiation. The aim was to cleanly remove the cathode while leaving intact all underlayers. Since an 80 $\mathrm{nm}$ thick white light emitting polymer (LEP) layer was in direct contact below the cathode, a visible wavelength (532 $\mathrm{nm}$ ) was chosen where both organic layers (LEP and PEDOT:PSS) as well as the ITO and barrier layers show negligible absorption, in contrast to the cathode. The cathode reflectivity is almost constant between 500-1000 $\mathrm{nm}$.

A series of single-pulse ablated craters with increasing pulse energy Ep on target was measured. Both the crater diameter and depth increased with Ep, as expected, due to the almost Gaussian intensity profile of the focussed spot.

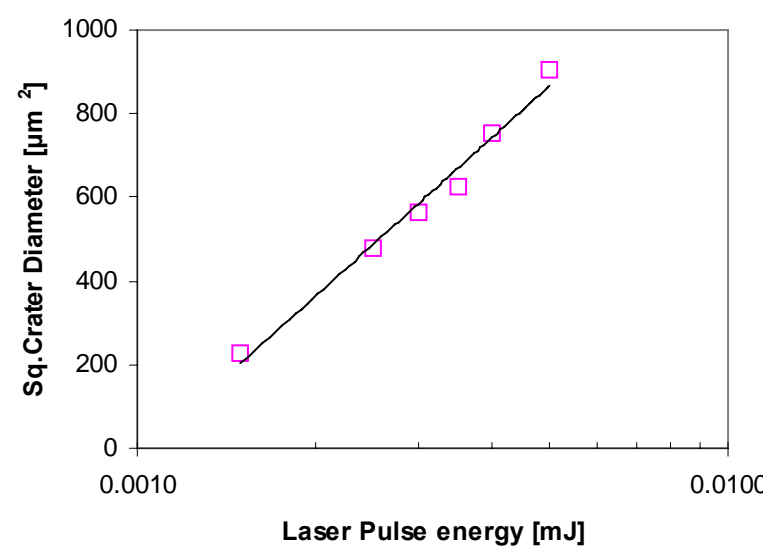

Fig. 5 Ablated crater area $v$ incident laser pulse energy for $532 \mathrm{~nm}, 10 \mathrm{ps}$ single pulse ablation of $\mathrm{Ba} / \mathrm{Al}$ cathode on OLED layers. The fitted curve slope reveals an ablation threshold of $95 \pm 9 \mathrm{~mJ} / \mathrm{cm}^{2}$ 


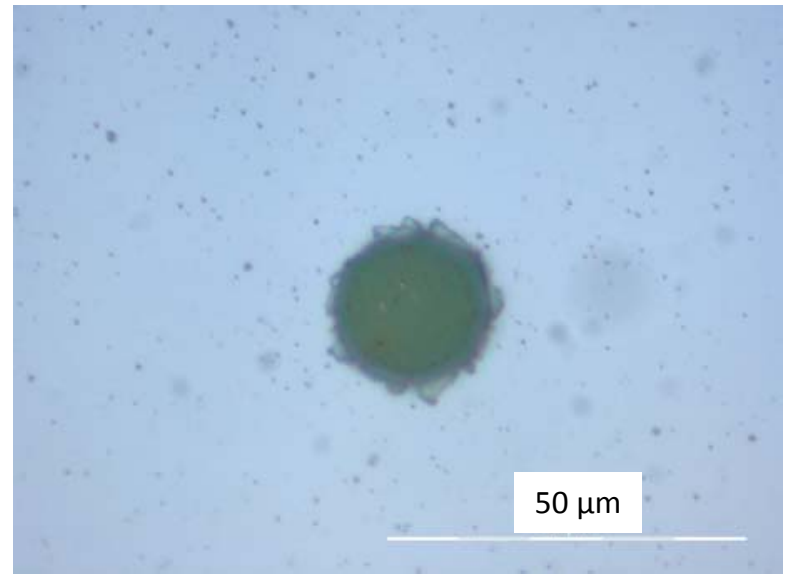

(a)

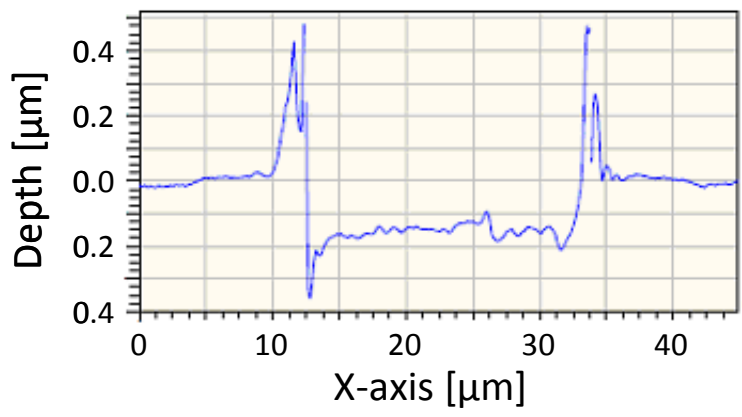

(b)

Fig. 6 (a) Optical microscope image of ps laser ablated cathode crater on LEP. Single pulse ablation, $532 \mathrm{~nm}$, $10 \mathrm{ps}, 2.5 \mu \mathrm{J}$; Bar $50 \mu \mathrm{m}$, (b) Depth profile of the ablated crater, depth $140 \mathrm{~nm}$. Scale $(\mathrm{X}): 2.5 \mu \mathrm{m} / \mathrm{div},(\mathrm{Y}): 25 \mathrm{~nm} / \mathrm{div}$.

By plotting therefore the crater squared diameter against the pulse energy (Fig.5), a single-pulse ablation threshold for cathode removal was estimated as $95 \pm 9$ $\mathrm{mJ} / \mathrm{cm}^{2}$ from the slope of the fitted curve.

Laser irradiation at fluences between $137-360 \mathrm{~mJ} / \mathrm{cm}^{2}$ removed the cathode layer only, resulting in a uniform flat floor and an intact LEP surface, allowing a relatively wide process window for cathode removal. A typical example of such laser patterned $\mathrm{Ba} / \mathrm{Al}$ cathode layer on the OLED stack is shown in figure 6 . The average fluence was 230 $\mathrm{mJ} / \mathrm{cm}^{2}$ irradiated with an estimated spot diameter at $1 / \mathrm{e}^{2}$ of $35 \mu \mathrm{m}$. This resulted in a crater diameter of $21.5 \mu \mathrm{m}$. One drawback with the current optical setup is the relatively rough crater boundaries obtained during rupturing from the non-uniform spatial irradiation. These irregular shaped edges are typically folded back towards the adjacent unirradiated surface, contributing to a rim elevation of 500 $\mathrm{nm}$ or more, which can be detrimental to further processing steps. Introducing a more uniform beam profile, comparing to the present Gaussian beam, might reduce this effect. Interestingly, there is hardly any newly introduced laser -induced microdroplets or other "thermal signature" debris redeposited around the crater. That is good evidence of a low temperature removal process and quite encouraging for OLED manufacturing as the presence of surface microparticles is linked to the introduction of black spots and faster degradation of the OLED device performance and lifetime.
At higher fluences $\left(\mathrm{F}>360 \mathrm{~mJ} / \mathrm{cm}^{2}\right)$, two or more layers were ablated simultaneously and the craters were deeper at the centre, due to the higher Gaussian intensity locally. Similarly, multiple pulse irradiation created deeper craters by removing the underlying organic layers also. In both cases the stepped ablation craters point to the deterministic character of the removal process. The craters remained sharp with clean edges even at the highest examined fluence of $825 \mathrm{~mJ} / \mathrm{cm}^{2}$.

Laser removal of metal films on polymer substrates is generally considered challenging, metal ablation being energetically more expensive with the potential to easily damage a sensitive underlayer. Unlike nanosecond UV ablation of metals [15] where the ablated films are blown off in a liquid phase via a substrate assisted explosive removal, picosecond laser patterning can permit precise metal removal with little, if any, melting involved and generally limited thermal characteristics depending on the chosen laser parameters [16] and quality of film contact.

An exact mechanism for the clean metal removal in this case still remains unclear. When the laser pulse duration is of the order or shorter than the electron-phonon thermalisation time, laser matter interaction is a two-step, non-equilibrium heating process. The incident laser energy is absorbed by electrons during excitation and soon after a portion is transferred to the lattice through electron-phonon coupling. Meanwhile the hot electrons diffuse deeper in the material extending the heated depth. Both thermal and stress confinement criteria will be satisfied using a $10 \mathrm{ps}$ laser pulse and the metal layer will therefore become highly compressed upon laser heating. At low fluences, a hot-electron blast force generates a strong shock wave early on that weakens the material. This is compounded by the thermal load from the subsequent lattice heating after the laser pulse which could generate enough thermoelastic stress to exceed upon relaxation an expected tensile strength for Al of $450 \mathrm{MPa}$ and result in film fracture [17]. Such a qualitative explanation can support the observed lack of cathode melting during low power picosecond laser patterning.

It can also be argued though that diffusing heat from the irradiated cathode can raise the LEP temperature. Assuming a slow "heat leaking" rate from the LEP film to the further underlying PEDOT:PSS film, it is plausible that this temperature rise could cause thermal expansion of the LEP surface or even some degassing of hot escaping products from a non-decomposed LEP film and generate enough pressure behind the metal film that could contribute to the detachment of the anyway softer cathode near the melting point. Permanent damage from swelling observed on the LEP surface upon cathode ablation at slightly higher fluences could point to such an ablation route. Further experimental evidence exploring the timescale of cathode ablation on a multilayered OLED could clarify the removal mechanism further.

\section{Summary}

Experimental evidence suggests that selective laser patterning of multilayered OLED stacks is enabled using picosecond lasers at visible and UV wavelengths. Transparent ITO anode films on inorganic barrier layers or 
metal cathode on organic OLED layers can be cleanly removed without damage to the underlying layers most likely by a stress-assisted low temperature ablation process.

\section{Acknowledgments}

The authors gratefully acknowledge financial support from EU program FP7 ICT-3 FAST2LIGHT, No: 216641 and IMEC (Ghent, BE), Coherent Scotland, (Glasgow, UK) and Microbridge Services Ltd (Cardiff, UK) for access to their lasers, metrology equipment and helpful discussions with Piet Bouten, Philips Research (Eindhoven, NL).

\section{References}

[1] S.R.Forrest, Nature, 428, (2004) 911-918

[2] D.Lidzey, M.Voight, C.Gibeler, A.Buckley, J.Wright, K.Boehlen, J.Fieret, R.Allott, Org. Electr. 6 (2005) 221

[3] Y.Ito,Y.Onodera, R.Tanabe, M.Ichihara, H.Kamada, Proc.SPIE 6458 (2007) 64580C.1

[4] R.Fardel, M.Nagel, F.Nuesch, T. Lippert, A,Wokaun, Appl.Phys.Lett., 91 (2007) 61103

[5] www.fast2light.org
[6] J.D.Affinito, M.E,Gross, C.A.Coronado, G.L.Graft, I,.N.Greenwell, P.M.Martin Thin Solid Films 290-291, (1996) 63

[7] G.Paltauf and P.E.Dyer, Chem.Rev. 103 (2003) 487

[8] E.Leveugle, D.S.Ivanov, L.V.Zhigilei, Appl. Phys. A 79, (2004) 1643

[9] P.B.Corkum, F.Brunel, N.Sherman, T.Srinivasan-Rao, Phys.Rev.Lett., 61 (1988) 2886

[10]Z.Lin, L.V.Zhigilei, V.Celli, Phys.Rev.B 77 (2008) 75133

[11] S.G.Koulikov and D.D.Dlott, J. Photoch. Photobiol A: Chem 145, (2001) 183

[12] www.Bizesp.com

[13] O.Yavas and M.Takai, J.Appl.Phys. 85 (1999), 4207

[14]D.Bauerle "Laser Processing and Chemistry" (Springer, Berlin 1996) p.128

[15]P.E.Dyer, D.M.Karnakis, P.H.Key, D.Sands, Appl.Surf.Sci., 109/110 (1997) 168

[16]J.Yang, Y.Zhao, N.Zhang, Y.Liang, M.Wang, Phys.Rev B., 76 (2007) 165430

[17] J.K.Chen, J.E.Beraun, C.L.Tham, Int.J.Eng.Sci. 42 (2004) 793

(Received: July 7, 2009, Accepted: November 17 ,2009) 